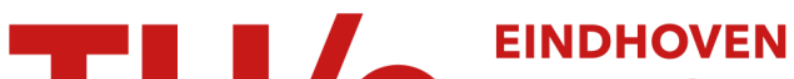 UNIVERSITY OF TECHNOLOGY
}

\section{Vortical motion in the head of an axisymmetric gravity current}

Citation for published version (APA):

Patterson, M. D., Simpson, J. E., Dalziel, S. B., \& Heijst, van, G. J. F. (2006). Vortical motion in the head of an axisymmetric gravity current. Physics of Fluids, 18(4), 046601-1/7. https://doi.org/10.1063/1.2174717

DOI:

10.1063/1.2174717

Document status and date:

Published: 01/01/2006

Document Version:

Publisher's PDF, also known as Version of Record (includes final page, issue and volume numbers)

Please check the document version of this publication:

- A submitted manuscript is the version of the article upon submission and before peer-review. There can be important differences between the submitted version and the official published version of record. People interested in the research are advised to contact the author for the final version of the publication, or visit the $\mathrm{DOI}$ to the publisher's website.

- The final author version and the galley proof are versions of the publication after peer review.

- The final published version features the final layout of the paper including the volume, issue and page numbers.

Link to publication

\section{General rights}

Copyright and moral rights for the publications made accessible in the public portal are retained by the authors and/or other copyright owners and it is a condition of accessing publications that users recognise and abide by the legal requirements associated with these rights.

- Users may download and print one copy of any publication from the public portal for the purpose of private study or research.

- You may not further distribute the material or use it for any profit-making activity or commercial gain

- You may freely distribute the URL identifying the publication in the public portal.

If the publication is distributed under the terms of Article $25 \mathrm{fa}$ of the Dutch Copyright Act, indicated by the "Taverne" license above, please follow below link for the End User Agreement:

www.tue.nl/taverne

Take down policy

If you believe that this document breaches copyright please contact us at:

openaccess@tue.nl

providing details and we will investigate your claim. 


\title{
Vortical motion in the head of an axisymmetric gravity current
}

\author{
M. D. Patterson, J. E. Simpson, and S. B. Dalziel \\ Department of Applied Mathematics and Theoretical Physics, Centre for Mathematical Sciences, \\ University of Cambridge, Wilberforce Road, Cambridge CB3 OWA, England \\ G. J. F. van Heijst \\ Fluid Dynamics Laboratory, Department of Physics, Eindhoven University of Technology, \\ PO Box 513, 5600MB Eindhoven, The Netherlands
}

(Received 11 February 2005; accepted 12 January 2006; published online 25 April 2006)

\begin{abstract}
A series of experiments that examine the initial development of an axisymmetric gravity current have been carried out. The experiments highlight the growth of a ring vortex that dominates the dynamics of the gravity current's early time propagation. In particular, the experiments show three distinct stages of early time development that have previously been described as the "initial phase" of a gravity current. The first phase of the early time development is dependent on the fractional depth of the lock release, followed by a secondary phase wherein the frontal speed is approximately constant and a third phase of reducing speed. The second phase of the gravity current's propagation comes to an abrupt end with the breakdown of the ring vortex at a clearly defined position. All of the experimental results show the development of a complex flow field where the generation and collapse of a ring vortex dominate the gravity current's early time propagation. The complexity of the flow field and the dependence of the propagation speed on the presence of the ring vortex in the head of the gravity current highlights the unsuitability of shallow-water modeling for axisymmetric lock releases at early times. (C) 2006 American Institute of Physics. [DOI: 10.1063/1.2174717]
\end{abstract}

\section{INTRODUCTION}

Gravity currents have been studied extensively in different configurations because of their importance to a large number of industrial and environmental situations. The general dynamics of a two-dimensional (2D) gravity current (a gravity current that propagates between parallel confines) are now thought to be well understood. Laboratory experiments ${ }^{1}$ have provided detailed observations of such flows. It was found that the flow arising after the release can be divided into a number of different phases. In the initial phase, the current develops and forms a raised head. Somewhat later, the gravity current reaches a second, self-similar phase. The behavior in this latter phase, in which the flow is governed by a balance between buoyancy and inertial forces, is more fully discussed in Grundy and Rottman. ${ }^{2}$ After sufficient time, the flow will enter a final phase, in which it is governed by a buoyancy-viscous balance that is well described by shallow-water theory.

Observations of axisymmetric experiments show that nearly all the spreading dense fluid is confined in the head of the gravity current leaving the area behind it almost clear of dense fluid. This behavior differs from a $2 \mathrm{D}$ gravity current where a long tail-like structure is observed. Figure 1(a) shows an axisymmetric gravity current from above, as observed in one of the Thorney Island trials, performed on an almost windless day (see McQuaid ${ }^{3}$ ). A similar structure can be seen in Fig. 1(b), which presents a plan-view photograph of a laboratory experiment of a fixed-volume saline release in a fresh-water ambient.

While the late time behavior of 2D and axisymmetric gravity currents are well understood, there are situations when the early time behavior of a gravity current is of interest. For example, the spread of a dense gas in a confined area due to the bursting of a chemical storage tank. Understanding and modeling the early time dynamics of the gravity current arising after such an accidental release is crucial for a better risk assessment. The depth and velocity profiles of the dense fluid for both the 2D and axisymmetric lock release configurations are well described away from the initial lock release by the numerical simulations based on the shallowwater approximation. ${ }^{4,5}$ Results from these shallow-water models show considerable differences between the predicted shape of the front for the distinct initial conditions. As with the experimental observations, numerical simulations show dense fluid from the lock release spreads at a relatively constant depth over the gravity current's length for the 2D case, while in the axisymmetric case, most of the dense fluid appears to become concentrated between the front and a backward-facing hydraulic jump; this jump is a result of post-rarefaction wave reflection from the origin and it propagates radially outward more rapidly than the front itself. Rottman and Simpson ${ }^{4}$ proposed that the catch-up of the backward-facing hydraulic jump is the mechanism that is responsible for the formation of the head of the gravity current. All of the work using shallow water simulations ignores vertical accelerations, viscous effects, and mixing between the fluids. Ungarish and Zemach ${ }^{6}$ provide a more complete overview of the modeling of slumping high Reynolds number gravity currents in two-dimensional and axisymmetric configurations.

The geometry of an axisymmetric gravity current can be defined in terms of the overall depth of the ambient fluid $(H)$, the depth of dense fluid in the lock release $\left(h_{0}\right)$, and the 


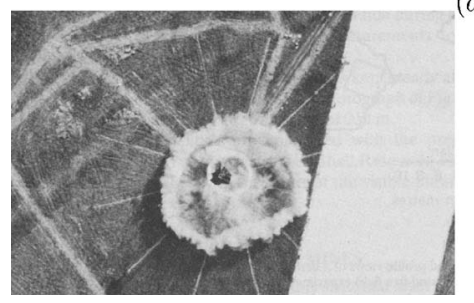

(a)

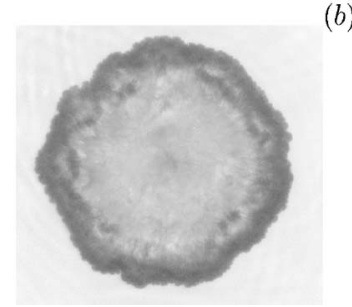

FIG. 1. (a) Aerial photograph of the spreading dense gas cloud in trial 8 of the Thorney Island field experiment (see Ref. 3). (b) Plan view of a laboratory experiment showing the radial spreading of a fixed volume of dense fluid released in a lighter fluid.

radial distance of the lock gate from the origin $\left(R_{0}\right)$. The flow can then be characterized by the reduced gravity $\left(g^{\prime}=\Delta \rho g / \rho\right)$, the fractional depth $\left(\phi=h_{0} / H\right)$, and the aspect ratio $\left(\gamma=h_{0} / R_{0}\right)$ of the releases. In this paper we examine the early time development of an axisymmetrically spreading gravity current in some detail, and in particular the effect of the fractional depth of the initial dense-fluid volume on the spreading characteristics. This study consists of laboratory experiments and numerical simulations, described in Secs. II and III, respectively. The main results of the experiments and the simulations are presented in Sec. IV. Some general conclusions are discussed in Sec. V.

\section{LABORATORY ARRANGEMENT}

Laboratory experiments have been carried out in a sector-shaped tank $2.35 \mathrm{~m}$ long, with a sector angle measuring approximately $10^{\circ}$ (see Fig. 2). In the first set of experiments, the sector tank was filled with fresh water (density $\rho_{A}$ ) to a depth $H=300 \mathrm{~mm}$. A fixed volume of denser fluid (with a density difference, $\Delta \rho=13.5 \mathrm{~kg} / \mathrm{m}^{3}$ ) was contained behind a vertical barrier at a radial distance $R=R_{0}$ and at a depth $h_{0}$, where $h_{0} \leqslant H$. The region of depth $H-h_{0}$ above the dense layer contained ambient fluid of density $\rho_{A}$. By lifting the barrier, the denser fluid is released into the widening part of the tank. For visualization purposes, the dense fluid was dyed with potassium permanganate. Illumination from behind using a diffuse light bank resulted in a sharply defined interface between the gravity current and the ambient fluid. The evolution of the resulting gravity current was recorded using a high resolution digital video camera (JAI model CV-M4+) with the movies saved to a computer.

An initial series of experiments were carried out for different values of $\phi$, in order to investigate the role of the fractional depth of the lock release on the general characteristics of the gravity current; see Table I for details. Four experiments are reported on, and the parameters were taken

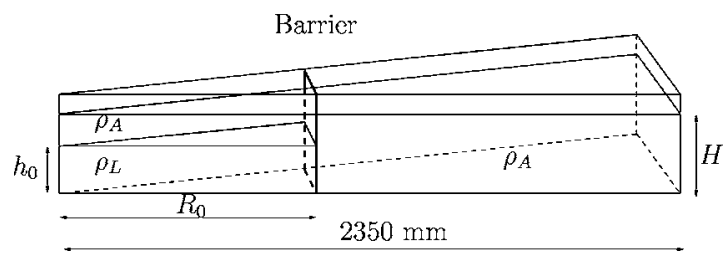

FIG. 2. Schematic diagram showing the sector tank.
TABLE I. Experimental details of the front position experiments.

\begin{tabular}{clll}
\hline \hline Experiment & $h_{0}(\mathrm{~mm})$ & $\phi$ & $\gamma$ \\
\hline 1 & 300 & 1.0 & 0.5 \\
2 & 220 & 0.733 & 0.366 \\
3 & 175 & 0.583 & 0.292 \\
4 & 90 & 0.3 & 0.15 \\
5 & 75 & 0.25 & 0.125 \\
6 & 300 & 1.0 & 0.5 \\
\hline \hline
\end{tabular}

as follows: $g^{\prime}=13.2 \mathrm{~cm} \mathrm{~s}^{-2}$, while the fractional depth $\phi$ and the aspect ratio $\gamma$ were varied between $0.25-1.0$ and $0.125-$ 0.5 , respectively.

A further investigation into the general dynamics of the axisymmetric gravity current is undertaken using particle image velocimetry (PIV) to examine the temporal and spatial development of the gravity current's head. Pattern matching algorithms developed by Dalziel $^{7}$ have been adapted for this purpose. For this set of experiments both the lock and the ambient fluid were seeded with a mixture of particles of differing densities. The mixture was composed of 150-212 $\mu \mathrm{m}$ Pliolite VT, 180-200 $\mu \mathrm{m}$ Pliolite VT-L and 200-250 $\mu \mathrm{m}$ Pliolite VTACH in approximately equal quantities. The Pliolite particles have a density range of $1002-1005 \mathrm{~kg} / \mathrm{m}^{3}$, their small size results a negligible volume of particles sedimenting out over the duration of the experiments. The flow was illuminated using a $5 \mathrm{~mm}$ vertical light sheet created by using a $300 \mathrm{~W}$ arc lamp with an integral parabolic dichroic reflector to collimate the light. The use of a light sheet allows three-dimensional (3D) motion to be filtered out as only the particles that remained in the light sheet are bright enough to be detected by the camera. Details of the PIV experiment described herein are listed in Table I (experiment 6).

\section{NUMERICAL MODELING}

The equations of motion describing the motion of a fluid of variable density are

$$
\begin{aligned}
& \mathbf{u}_{t}+(\mathbf{u} \cdot \nabla) \mathbf{u}=\frac{1}{\rho}\left(-\nabla p+\mu \nabla^{2} \mathbf{u}+\mathbf{H}_{u}\right), \\
& \rho_{t}+(\mathbf{u} \cdot \nabla) \rho=0, \\
& \nabla \cdot \mathbf{u}=0,
\end{aligned}
$$

where $\mathbf{u}=(u, v, w), \rho, \mu$, and $p$ represent the velocity, density, dynamic viscosity, and pressure, respectively. External forces are represented by $\mathbf{H}_{u}=(0,0,-\rho g \hat{z})$.

Three-dimensional numerical flow simulations have been carried out with the monotone-integrated large-eddy simulation (MILES) code described in Almgren et al. ${ }^{8}$ The code used incorporates an incompressible Navier-Stokes solver where the equations of motion (1)-(3) are solved using a second-order upwind Godunov method for the advection step, Crank-Nicolson discretization of the viscous and diffusive terms and a variable density approximate projection to impose the divergence constraint.

The main difference between this MILES based code and 
other Navier-Stokes solvers is that there are no explicit closure schemes applied to solve for turbulence. Oran and Boris ${ }^{9}$ provide a general overview of MILES. The simulations are used to resolve the macroscopic scales in the problem and it is assumed that the flux-limiting monotone algorithms used for solving the set of coupled equations have a highfrequency filter that inherently maintains physically reasonable behavior near the grid scale cutoff defined as the domain length/number cells.

\section{Numerical simulations}

The Navier-Stokes equations for a 3D flow are computationally expensive. A straightforward method of reducing the computational effort for an axisymmetric flow is to model the flow as a 2D radial Navier-Stokes (2DRNS) problem. Numerically, this implies that we solve the NavierStokes equations on a 2D Cartesian grid and include source terms that allow for the radial spread of the fluid. The majority of the numerical work discussed here sets out to model the experimental results using a 2DRNS solver.

Observations from the experimental study have, however, revealed that the flows are intrinsically $3 \mathrm{D}$, with a vortical flow structure at the front, and with lobes and clefts being clearly observed to form as the gravity current propagates outwards. Two-dimensional radial flow models cannot account for the formation of lobes and clefts or the breakdown of vortical structures due to these 3D instabilities.

Fully 3D calculations on a Cartesian grid have therefore been carried out to determine when and where 3D effects become important in the gravity current's front propagation. The 3D simulations are carried out on a $256 \times 256 \times 32$ Cartesian grid and are compared with a 2 DRNS on a $256 \times 32$ grid. The initial conditions can be arranged to reduce the effective grid size in 3D simulation to a quarter cylinder with its center on the origin. This is equivalent to modeling a full cylindrical release at $512 \times 512 \times 32$; however, it results in the same grid scale cutoff as the 2DRNS.

For 2D and 3D simulations where we do not explicitly define a viscosity (which will from now on be referred to as "inviscid") we set free-slip boundary conditions along all boundaries; the 2D simulations where we explicitly define viscosity are performed with a free-slip boundary condition enforced on the free surface and no-slip boundary conditions at the other boundaries. While for the 3D viscous simulations the horizontal base and the two exterior boundaries not in contact with the origin have no-slip conditions applied, freeslip conditions are enforced at the other boundaries.

\section{RESULTS}

\section{A. The development of an axisymmetric gravity current}

The development of a full-depth gravity current $(\phi=1.0)$ can be observed in Figs. 3(a)-3(e), which present experimental snapshots of the flow, together with the corresponding density plots from a 2DRNS numerical simulation.

The figures clearly show the head of the gravity current starting to develop shortly after the lock release [Fig. 3(a)].

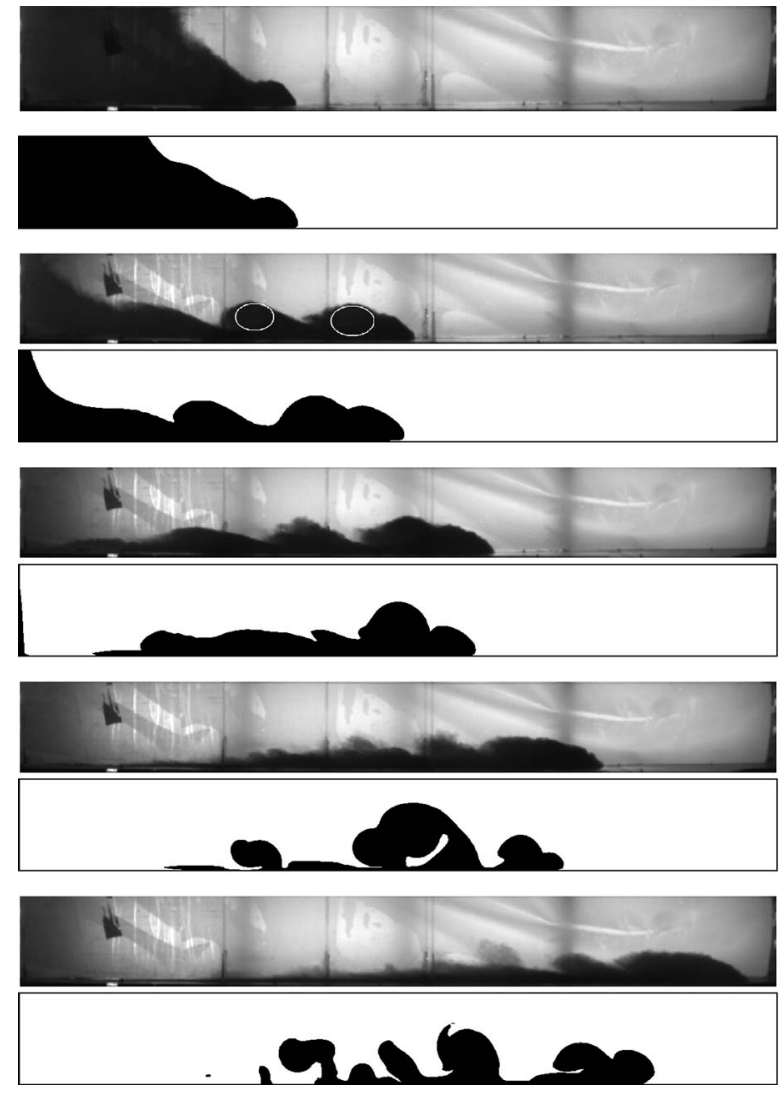

(a)

$(c)$

$(d)$

(e)

FIG. 3. Experimental (upper picture) and numerical (lower picture) results showing the evolution of the radially spreading gravity current for an axisymmetric lock release. Experiment 1, with $\phi=1.0, g^{\prime}=13.2 \mathrm{~cm} / \mathrm{s}^{2}$ for times $t=2.59 \mathrm{~s}(\mathrm{a}), 6.19 \mathrm{~s}(\mathrm{~b}), 8.70 \mathrm{~s}(\mathrm{c}), 12.29$ (d), and $17.81 \mathrm{~s} \mathrm{(e)}$.

During the collapse of the dense fluid volume, vorticity is generated mainly at the interface between the lighter and denser fluids, and there is also a lesser production of vorticity at the no-slip boundaries. The resulting gravity current head appears to exhibit typical features of a shear interface. However, as the gravity current's head is advected by the radially spreading fluid, vorticity accumulates near the front, contributing to the formation of a gradually expanding leading ring vortex. A secondary ring vortex is also generated and the locations of the ring vortices are marked as white ellipses in Fig. 3(b). The secondary vortex ring forms behind the primary ring, it is not shed from the front of the gravity current as it propagates forward. Vortex stretching appears to stabilize the rings [see Fig. 3(c)], and these ring vortices become more intense. In a 2D gravity current coherent vortical motion breaks down rapidly. As the rate of stretching decreases (with increasing distance from the origin) the vortical flow can no longer be maintained [Fig. 3(d)] and the ring vortex collapses abruptly. The front then continues to propagate outward without any clearly defined vortical motion [Fig. 3(e)]. Comparison of the experimental and numerical results shows an excellent agreement for the initial stages of the flow (before vortex breakdown), with the front position and the form of the gravity current being accurately predicted, the 2DRNS does not allow for the breakdown of vortical motion and does not model the flow well after the breakdown of the ring vortex. 


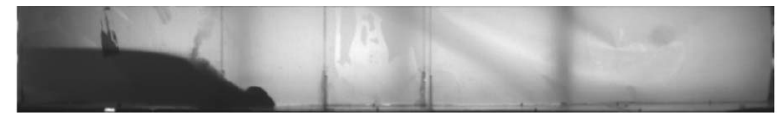

(a)

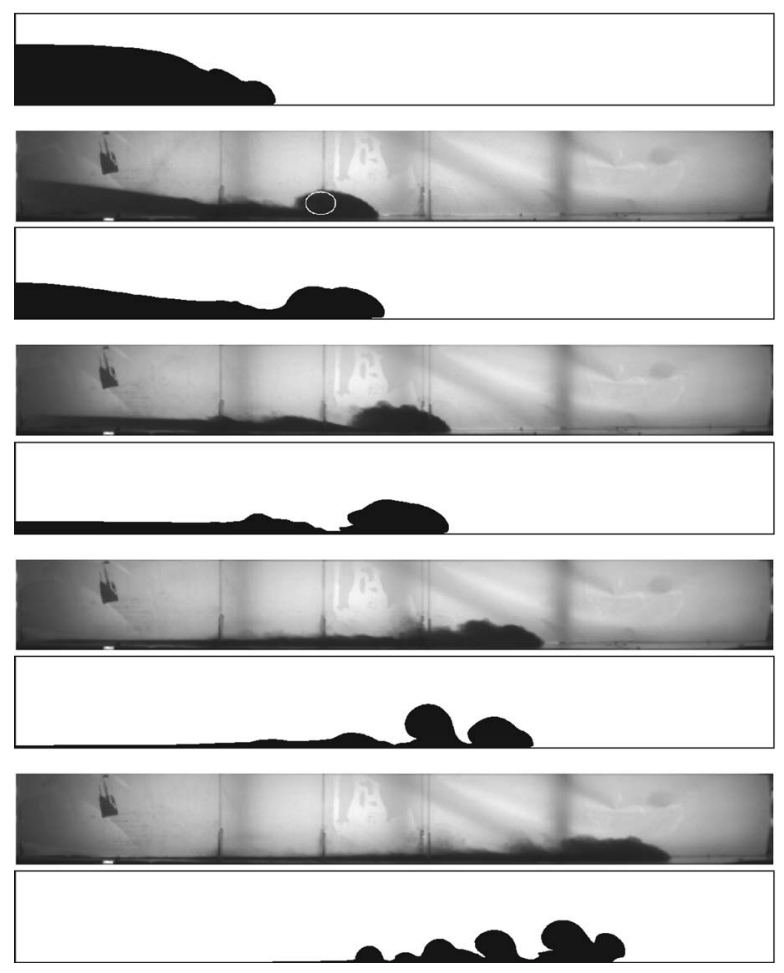

(b)

(e)

FIG. 4. Same as Fig. 3, showing results from experiment 3, with $\phi=0.583$ and $g^{\prime}=13.2 \mathrm{~cm} / \mathrm{s}^{2}$ for times $t=2.59 \mathrm{~s}$ (a), $6.19 \mathrm{~s}(\mathrm{~b}), 8.70 \mathrm{~s} \mathrm{(c),}$ 12.29 (d), and $17.81 \mathrm{~s} \mathrm{(e)}$.

The results in Figs. 4(a)-4(e) show the evolution of the spreading dense fluid for the case where $\phi=0.58$. In contrast to the previous case, now only a single ring vortex front is visible and the velocities inside the ring vortex in the frontal bulge are smaller than for the release of a full-lock-depth volume $(\phi=1.0)$. As in the previous results, the ring vortex is marked in Fig. 4(b). Again, the numerical simulation accurately predicts the position and the shape of the gravity current up to the point where the vortex breaks down.

\section{B. Front propagation}

While Figs. 3(a)-3(e) and 4(a)-4(e) clearly show the general characteristics of axisymmetrically spreading gravity currents, further insight into the development of a gravity current may be gained by the examination of its front propagation speed. We distinguish here three different stages in the gravity current's early time propagation: a short initial stage of constant velocity, a secondary phase of a higher constant velocity, and a third phase of reducing velocity. These are described below in the following sections. Note that due to tank size limitations, we do not consider the late time buoyancy-viscous balance stage.

\section{Initial phase}

Improved methods of recording and processing the experimental data allow for a more detailed examination of the frontal position at earlier times than was previously feasible. (a)

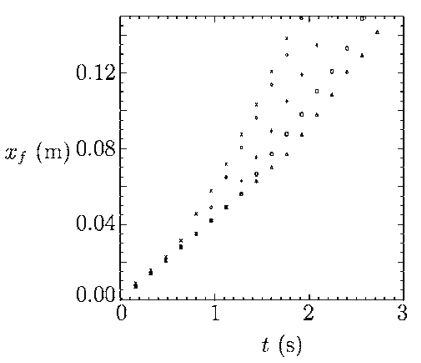

(b)

FIG. 5. (a) The position of the front plotted against time for the initial phase of the gravity current. Experiment $1(\times), 2(\diamond), 3(+), 4(\square)$, and $5(\triangle)$. (b) The position of the breakdown of the initial phase plotted against the initial fractional depth $\phi$.

The results for the first series of experiments are shown in Fig. 5, where the reduced gravity $\left(g^{\prime}=13.2 \mathrm{~cm} / \mathrm{s}^{2}\right)$ is kept at the same value for all the gravity currents. The results clearly indicate the presence of a short initial phase in which the front travels outward at a constant speed; in all cases shown, the initial propagation speed is the same and not dependent on the fractional depth. The initial constant velocity of the front lasts for 0.8 to $2.0 \mathrm{~s}$, after which a sudden acceleration of the front is observed. The experimental data clearly show that the time the flow spends in this initial phase depends solely upon the fractional depth $\phi$, of the lock release. Larger depth lock releases move out of the initial constant velocity phase more rapidly than the shallower releases.

As far as we are aware, results of this accuracy capturing the initial stages of motion have not been previously reported, neither for 2D nor for axisymmetric gravity currents.

\section{Secondary and tertiary phases}

Figures 6(a)-6(d) show the frontal position plotted against time for the set of experiments detailed in Table I. Each plot contains two least-squares best-fit lines applied to the data highlighting the secondary and tertiary phases of the gravity current's propagation. These results clearly reveal that the secondary phase (when the ring vortex is present above the head of the gravity current) of the flow is of constant speed, where the speed is a function of $\phi$. Although the experiments described above cover a large range of fractional depths, the best-fit lines can clearly be seen to intersect at the same position within experimental error bounds, indicating a possible change of regime in the area of intersection of the two best-fit lines. This position is approximately $1.7 R_{0}$, and appears to be constant for all experiments. Also included in the figures are two 2DRNS numerical simulations, one an inviscid simulation and the other a viscous flow $\left(\nu=10^{-2} \mathrm{~cm}^{2} / \mathrm{s}\right)$, both on a $256 \times 32$ grid. Analysis of these results show that the 2 DRNS model is capable of accurately predicting the position of the front up to approximately $u_{f} / R_{0} \approx 1.7$.

\section{Laboratory work and full scale experiments}

A comparison of the position where transition between the secondary and tertiary phases $R_{*}=x_{f} / R_{0}$ for all of the experimental and numerical work is shown in Fig. 7. A trial 
(a)
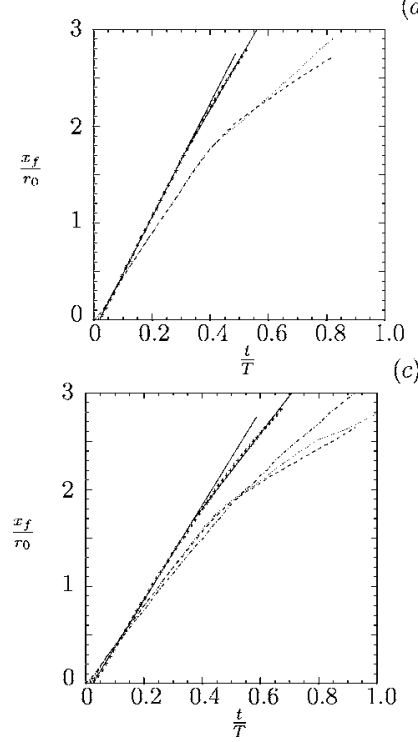

(c)

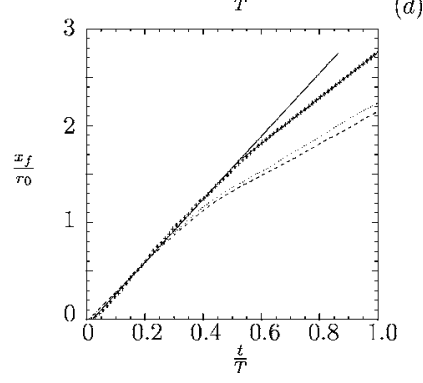

(b)

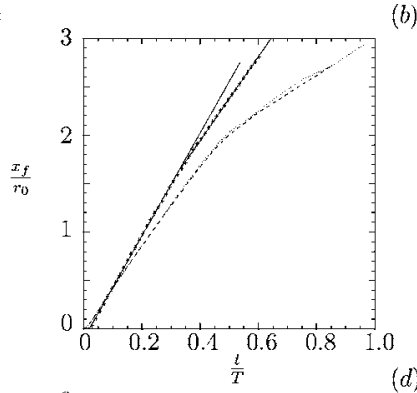

d)

FIG. 6. (a)-(d) Frontal position plotted against time for experiments 1, 2, 3, and 5. The experimental data $(+)$ is plotted with two first-order least-square fits (-). Numerical results for corresponding initial conditions on a 512 $\times 64$ grid for a 2DRNS are displayed for the inviscid (- -) and viscous (..) $\mu=10^{-2} \mathrm{~cm}^{2} / \mathrm{s}$ cases. Graph (c) also shows a result for a fully $3 \mathrm{D}$ computation (.-.).

from the Thorney Island field experiment $\left(\right.$ Picknett $\left.^{10}\right)$ has also been selected (trial no. 8 has been used because it was carried out on an almost windless day). Figure 1(a) shows an aerial photograph of the spreading dense gas volume. In this particular experiment the density of the dense gas (a mixture of air and freon) was $\rho=2.0 \mathrm{~kg} / \mathrm{m}^{3}$, compared with $1.2 \mathrm{~m} / \mathrm{s}^{3}$ for air. Unlike the laboratory experiments, the flow in the field experiment is non-Boussinesq. The initial volume of dense gas was approximately cylindrical, with a radius $R_{0}=7 \mathrm{~m}$ and a height $h_{0}=13 \mathrm{~m}$. Given the vertical dimension of the atmosphere, this situation can be considered as $\phi=0$. The spreading of the dense gas shows the same features as observed in the laboratory experiments on a much smaller scale; viz., a spreading at constant speed, followed by an abrupt change to the next phase. The scaled position $R$ * at which the transition occurs in the field experiments was

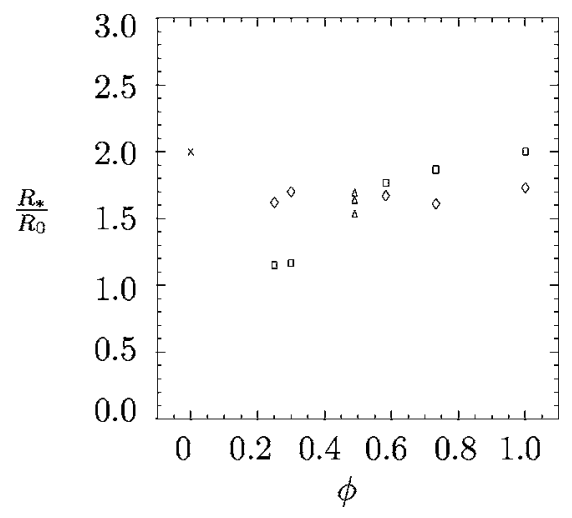

FIG. 7. Plot of the measured values of $R_{*} / R_{0}$ as a function of the fractional depth, $\phi$, for the laboratory experiments $(\diamond)$, trial no. 8. of the Thorney Island field experiment $(\times)$, and the numerical simulations $(\square)$. approximately $R_{*}=2$; these data have been obtained from an array of approximately 20 sensors spaced over a square kilometer. In the laboratory experiments, the transition point from secondary to tertiary phases was found to have an average value of $R_{*}=1.7$. Even though the scales of the field and laboratory experiments and the Reynolds numbers at which they propagate are vastly different, the values found for the transition point are comparable.

\section{Azimuthal instabilities in the vortex structure}

The development and propagation of a ring vortex in the head of the axisymmetric gravity current has been shown to be a fundamental feature of the early stages of the gravity current's propagation.

The study of Swearingen et al. ${ }^{11}$ of the generic behavior of a ring vortex impacting a solid boundary provides an interesting insight into one of the physical effects that may contribute to the collapse of the ring vortex and the transition from the secondary to the tertiary phases of the flow.

Swearingen et al. show using direct numerical simulations and comparison with experiment that as a ring vortex impacts with a wall at normal incidence a boundary layer forms which undergoes separation and roll-up to form a secondary ring vortex of opposite vorticity. The secondary ring can develop azimuthal instabilities which grow rapidly because of vortex stretching and tilting in the presence of the mean straining field generated by the primary ring vortex. The ring vortex tends to undergo this transition at $x_{f} / R_{0}$ $\approx 1.7$, which is identical to the position of transition found in these experiments.

\section{Particle image velocimetry: Full depth gravity current $(\phi=1)$}

The details of a PIV experiment for a full depth $\phi=1$ gravity current are presented in Figs. 8(a)-8(d) (supplemental video footage is available by clicking on the figure in the online article). The results provide significant insight into the different stages of the gravity current and how the ring vortices form and subsequently decay.

When the cylindrical volume of dense fluid begins to collapse, it spreads radially into the less dense ambient fluid. During this collapse the fluid released from the lock is accelerated in a downward radially expanding direction, while the less dense ambient fluid is accelerated upward and towards the origin. A shear layer associated with the interface between the dense downwardly accelerating fluid and the lighter upwardly accelerating ambient fluid can be seen in Fig. 8(a). As the gravity current continues to propagate outward at constant speed the shear layer extends until a perturbation to the shear layer (caused by the slope of the interface not being constant) causes two ring vortices to form, the instability is effectively a Kelvin-Helmholtz instability in a radially expanding domain. The ring vortices propagate outwards [Fig. 8(b)], entraining ambient fluid from above and dense fluid from the tail. As the gravity current continues outward the ring vortices continue entraining fluid [Fig. 8(c)] until a stage that they become unstable and collapse, after which the third stage of the gravity current sets in [Fig. 8(d)]. 

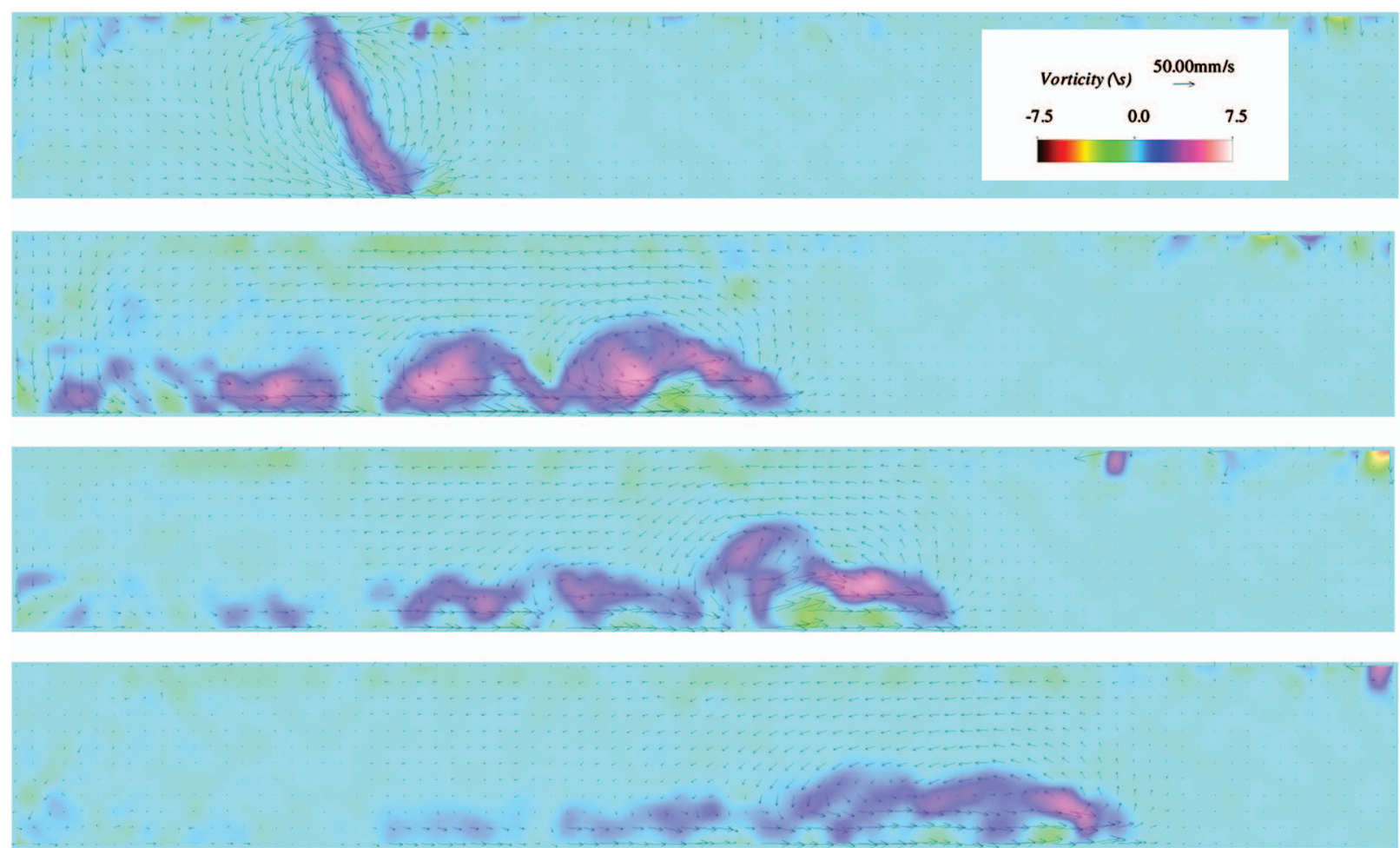

FIG. 8. (Color) (a)-(d) PIV and vorticity results derived from experiment 6 at times $t / T=0.055,0.38,0.53$, and 0.71 (enhanced online).

Previous work ${ }^{12}$ has examined the steady state flow structure within the head of a 2D gravity current using particle tracking velocimetry. The results of that study are in agreement with numerical simulations ${ }^{13}$ and show that velocities just behind the head of the gravity current were typically $20 \%$ higher than the speed of the advancing front. The flow structure within the head appears to involve dense fast moving fluid from the tail entering the head and then recirculating upwards when it reaches the front. The experimental results for the axisymmetric case show that flow in the lower part of the head of the gravity current is opposite in rotational direction to the $2 \mathrm{D}$ gravity current; this motion is due to the presence of the strong ring vortices and does not persist when the ring vortex has collapsed. Flow velocities in the tail of the gravity current have been observed to be up to $40 \%$ of that of the front. Figure 9 shows a schematic drawing of the head of a 2D and an axisymmetric gravity current, the differences in the velocity fields within the gravity currents are clearly indicated. Inspection of the experimental results shows that there does not appear to be a significant transfer of mass between the ring vortices and the head of the gravity current, although both draw mass from the collapsing lock release.

Analysis of the PIV experimental work provides sufficient information to propose a new mechanism (distinct from the azimuthal instability discussed in Sec. IV B 4) for the development and breakdown of the ring vortex at the head of an axisymmetric gravity current. As the gravity current propagates forward, vorticity is generated at the density interface and becomes concentrated in ring-shaped vortex structures. A ring vortex at the front builds in strength while being stretched (due to radial expansion), thus remaining identifiable as a coherent structure on the top rear of the gravity current's head. While the gravity current head propagates forward, the vortex ring is gradually left behind the head of the gravity current. At this stage the ring vortex cuts off the supply of dense fluid to the head of the gravity current, and all the fast moving fluid from the tail is then entrained into the ring vortex. The increased supply of dense fluid to the ring vortex provides a perturbation sufficiently large to destroy any coherent vortical motion. At the same time constant supply to the head of the gravity current is impeded, which causes the decrease of the frontal velocity. Inspection of these results shows that the leading ring vortex breaks down completely at approximately $R_{*}=1.7 R_{0}$, which corresponds with the location where a change in frontal velocity has been observed.
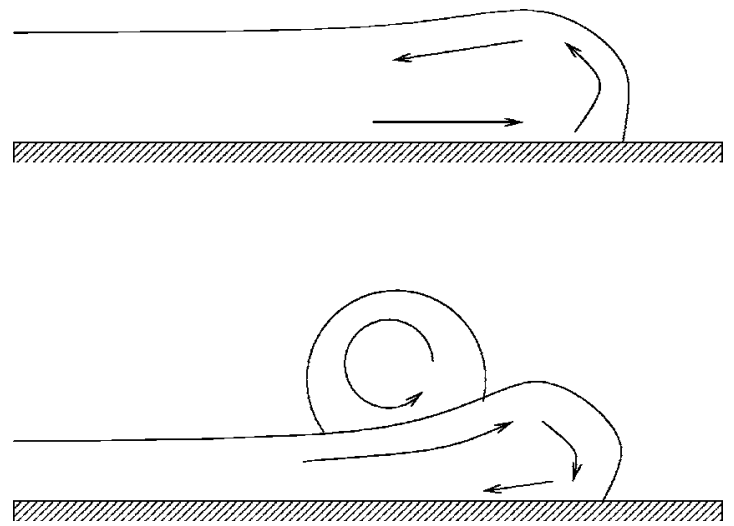

FIG. 9. A schematic drawing of the velocity field within the head of a $2 \mathrm{D}$ and an axisymmetric gravity current. 


\section{DISCUSSION AND CONCLUSIONS}

The early time development of an axisymmetric high Reynolds number gravity current from a fixed-volume release of dense fluid into a less dense ambient fluid has been examined using a variety of experimental techniques. The experimental results have been compared to a numerical study based on a monotone-integrated large-eddy simulation (MILES).

The experimental results clearly reveal the existence of three distinct phases in the flow evolution: (i) a short initial phase that is dependent upon the fractional depth $(\phi)$ of the lock, (ii) a constant velocity is then observed with a clearly defined ring vortex structure present above the spreading head of the gravity current, and finally (iii) a third phase in which the front appears to spread at constant speed until the end of the tank. There is no definable coherent vortical structure in this later stage. The limited tank length does not allow for a study of the gravity current's behavior at late times, although it is entirely reasonable to assume that a fourth phase will occur in which the flow is governed by a viscousbuoyancy balance.

The change from the secondary to the tertiary phase is easily observed by examination of the experimental data. When the ring vortex reaches a critical radius $R_{*} \approx 1.7 R_{0}$, the ring vortex collapses abruptly. Two distinct phenomena are identified as potential mechanisms for this breakdown. First, as the ring vortex is advected outward, it rolls of the top rear of the head of gravity current towards the base of the tank. The ring vortex then begins to interact with the base of the tank and cuts off the supply of dense fluid to the head of the gravity current; all the fast moving fluid from the tail is then entrained into the ring vortex. The increased supply of dense fluid to the ring vortex creates a significant perturbation to the vortical motion and results in the destruction of the ring vortex. The second potential cause for the breakdown of the ring vortex (which can also be observed for a ring vortex propagating in a homogeneous fluid ${ }^{11}$ ) is that a ring vortex will become unstable due to azimuthal instabilities caused by interaction of the ring vortex and the solid tank floor. This instability is due to the interaction of the primary ring vortex and the base of the tank, which results in the generation of a secondary ring vortex of opposite rotational direction that wraps around the primary vortex. As with the axisymmetric gravity currents discussed here, a ring vortex in a homogeneous fluid will become unstable at $\left(R_{*} \approx 1.7\right)$. While it is clear from observation of the experimental data that the first mechanism occurs, it is not so clear that the growth of the azimuthal instabilities in a secondary ring vortex develop sufficiently to destroy the primary ring vortex in the gravity current experiments described here. The growth of the azimuthal instabilities does appear to provide a suitable mechanism to explain the position of the transition. For all experiments, the position of $R_{*}$ is approximately constant.

Previous investigations into axisymmetric gravity currents have based their analyses on shallow-water models; i.e., ignoring any vertical accelerations, viscous effects, and mixing between the fluids. Although these calculations give a reasonable description of the overall behavior of the flow, the vortical motion near the front is not captured. If the early time behavior of the gravity current is of interest, an alternate modeling strategy is necessary. Examination of the 2DRNS and $3 \mathrm{D}$ results has revealed that the relative magnitude of the vertical velocities compared to the horizontal ones in the head of the gravity current are typically of the order $40 \%-$ $60 \%$ of that of the horizontal velocities. It should be emphasized that although these results show that it may be unsuitable to use a shallow-water model for early time modeling of a gravity current, it is still reasonable to assume that a shallow-water model should be capable of modeling the flow field accurately for late times after the large scale coherent vortical motion has broken down.

\section{ACKNOWLEDGMENTS}

One of the authors (M.D.P.) is supported by the Natural Environment Research Council on Grant No. NER/A/S/ 2001/01132. The code used is the incompressible, variabledensity Navier-Stokes solver written at the Center for Computational Sciences and Engineering, Lawrence Berkeley National Laboratory.

${ }^{1}$ J. E. Simpson, Gravity Currents in the Environment and the Laboratory (Cambridge University Press, Cambridge, 1997), 2nd ed..

${ }^{2}$ R. E. Grundy and J. W. Rottman, "The approach to self-similarity of the solutions of the shallow-water equations representing gravity-current releases,” J. Fluid Mech. 156, 39 (1985).

${ }^{3}$ Heavy Gas Dispersion Trials at Thorney Island: Proceedings of a Symposium Held at the University of Sheffield, Great Britain, 3-5 April 1984, edited by J. McQuaid (Elsevier, New York, 1985).

${ }^{4}$ J. W. Rottman and J. E. Simpson, "Gravity currents produced by instantaneous releases of a heavy fluid in a rectangular channel," J. Fluid Mech. 135, 95 (1983).

${ }^{5}$ J. W. Rottman and J. E. Simpson, "The initial development of gravity currents from fixed-volume releases of heavy fluids," in Atmospheric Dispersion of Heavy Gases and Small Particles, edited by G. Ooms and H. Tennekes, Proc. IUTAM Symposium (Springer-Verlag, Dordrecht, 1984).

${ }^{6} \mathrm{M}$. Ungarish and T. Zemach, "On the slumping of high Reynolds number gravity currents in two-dimensional and axisymmetric configurations," Eur. J. Mech. B/Fluids 2, 358 (2005).

${ }^{7}$ S. B. Dalziel, Digiflow User Guide, version 1.0 (DL Research Partners, Cambridge, U.K., 2005); http://www.damtp.cam.ac.uk/lab/digiflow/

${ }^{8}$ A. S. Almgren, J. B. Bell, and W. G. Szymczak, "A numerical method for the incompressible Navier-Stokes equations based on an approximate solver," SIAM J. Sci. Comput. (USA) 24, 71 (1996).

${ }^{9}$ E. S. Oran and J. P. Boris, Numerical Simulation of Reactive Flow, 2nd ed. (Cambridge University Press, New York, 2001).

${ }^{10}$ R. G. Picknett, "Field experiments on the behavior of dense clouds," Tech. Rep., Report No. Ptn IL 1154/78, CDE Contract Report to HSE-UK (1978).

${ }^{11}$ J. D. Swearingen, J. D. Crouch, and R. A. Handler, "Dynamics and the stability of a vortex ring impacting a solid boundary," J. Fluid Mech. 297, 1 (1995).

${ }^{12}$ L. P. Thomas, S. B. Dalziel, and B. M. Marino, "The structure of the head of an inertial gravity current determined by particle-tracking velocimetry," Exp. Fluids 34, 708 (2003)

${ }^{13}$ C. Härtel, E. Meiburg, and F. Necker, "Analysis and direct numerical simulation of the flow at a gravity-current head. Part 1. Flow topology and front speed for slip and non-slip boundaries," J. Fluid Mech. 418, 189 (2000). 